\title{
Analysis of a high-resolution optical wave-front control system ${ }^{\text {is }}$
}

\author{
E.W. Justh ${ }^{\mathrm{a}}$, P.S. Krishnaprasad ${ }^{\mathrm{b}, *}$, M.A. Vorontsov ${ }^{\mathrm{c}}$ \\ ${ }^{a}$ Institute for Systems Research, University of Maryland, College Park, MD 20742, USA \\ b Institute for Systems Research, Department of Electrical and Computer Engineering, University of Maryland, College Park, MD 20742, USA \\ ${ }^{\mathrm{c}}$ Intelligent Optics Laboratory, US Army Research Laboratory, Adelphi, MD 20783, USA
}

Received 27 September 2001; received in revised form 23 August 2003; accepted 6 February 2004

\begin{abstract}
We consider the formulation and analysis of a problem of automatic control: correcting for the distortion induced in an optical wave front due to propagation through a turbulent atmosphere. It has recently been demonstrated that high-resolution optical wave-front distortion suppression can be achieved using feedback systems based on high-resolution spatial light modulators and phase-contrast techniques. We examine the modeling and analysis of such adaptive optic systems, and show that under certain conditions, the nonlinear dynamical system models obtained are gradient systems (with energy functions that also serve as Lyapunov functions). These gradient systems (employing fixed phase-contrast sensors) serve as a starting point for understanding the design of practical high-resolution wave-front correction systems, in which the phase-contrast sensor itself is subject to control.
\end{abstract}

(C) 2004 Elsevier Ltd. All rights reserved.

Keywords: Adaptive optics; Wave-front control; Nonlinear stability; Lyapunov function

\section{Introduction}

Correcting for the distortion induced in an optical wave front due to propagation through a turbulent atmosphere can be formulated as problem of automatic control. Thermal gradients in the air produce refractive-index variations experienced by light which passes through it, leading to wave-front distortion (see Roggemann \& Welsh, 1996 for further discussion of the physics). Wave-front correction is achieved by applying (e.g., using an array of micromirrors) compensating distortions to produce net null distortion. Adaptive

\footnotetext{
This paper was not presented at any IFAC meeting. This paper was recommended for publication in revised form by Associate Editor Henk Nijmeijer under the direction of Editor Hassan Khalil. This research was supported in part by Army Research Office under ODDR\& E MURI97 Program Grant No. DAAG55-97-1-0114 to the Center for Dynamics and Control of Smart Structures (through Harvard University), and under ODDR\& E MURI01 Program Grant No. DAAD19-01-1-0465 to the Center for Communicating Networked Control Systems (through Boston University), and by the National Science Foundation Learning and Intelligent Systems Initiative Grant CMS9720334.

* Corresponding author. Fax: +1-301-314-9920.

E-mail addresses: justh@isr.umd.edu (E.W. Justh), krishna@isr.umd.edu (P.S. Krishnaprasad), mvorontsov@arl.army.mil (M.A. Vorontsov).
}

optics is the discipline concerned with feedback compensation of wave-front distortion in real time. The resolution and system bandwidth required for wave-front correction depends on the optical wavelength and on the strength of turbulence.

In high-resolution adaptive optics, the wave-front correction is provided by a liquid-crystal spatial light modulator (LC SLM) or by a microelectromechanical (MEMS) micromirror SLM. Although the physics of LC SLMs and MEMS micromirror SLMs is quite different, they serve the same function in an adaptive optic system: each consists of an array of independently actuated square pixels (where a pixel is a single piston-driven micromirror or a small square region of liquid crystal molecules driven by a single electrode), capable of imparting an electronically controlled, spatially varying phase shift (or wave-front change) to an optical beam. For high-resolution adaptive optics, the number of degrees of freedom (i.e., the number of SLM pixels) is $>10^{4} / \mathrm{cm}^{2}$, and the actuator stroke should be on the order of an optical wavelength. The pixel size (i.e., the length of each side of the pixel) needs to be at least 20 times the optical wavelength (so that fringing effects at the pixel edges can be neglected), and for practical reasons, it is generally desirable to keep the overall SLM area on the 


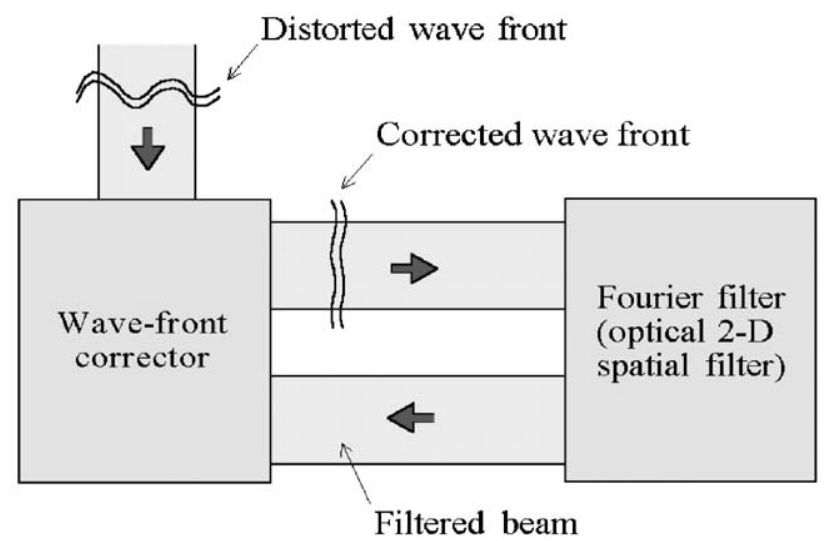

Fig. 1. High-resolution wave-front control system block diagram. The distorted beam is modulated by the wave-front corrector to produce the corrected beam, which is then filtered by the spatial Fourier filter. The wave-front corrector uses feedback from the Fourier filter.

order of a few square centimeters in size. LC and MEMS SLMs suitable for high-resolution adaptive optics have been developed only recently (Serati, Sharp, Serati, McKnight, \& Stockley, 1995; Horenstein, Bifano, Pappas, Perreault, \& Krishnamoorthy-Mali, 1999).

However, in addition to the devices, there is also a need for control laws which scale appropriately in this high-resolution, high-speed regime. Suitable control laws based on high-resolution SLMs and phase-contrast techniques have recently been demonstrated (Vorontsov, Justh, \& Beresnev, 2001; Justh, Vorontsov, Carhart, Beresnev, \& Krishnaprasad, 2001). Our focus here is on the modeling and analysis of these systems (Justh, Krishnaprasad, \& Vorontsov, 2000a; Justh \& Krishnaprasad, 2001).

In the next section we briefly review the high-resolution wave-front control system architecture and its origins. In Section 3, we introduce the mathematical models and analyze them. In Section 4, we digress from the analysis to clarify certain practical issues and provide context for the mathematical work. In Section 5, we consider the wave-front control system as a wave-front estimator. Concluding remarks appear in Section 6.

\section{High-resolution wave-front control system}

The general feedback system architecture we consider is shown in Fig. 1. The distorted beam enters the wave-front corrector, where (as shown in Fig. 2) a high-resolution SLM modulates the wave front with the objective of cancelling the distortion. (For clarity of the discussion, all beams are assumed to be monochromatic, although much of the analysis can be generalized to multi-wavelength beams or coherent white light.) The corrected beam is then filtered using a two-dimensional spatial Fourier filter, and the resulting filtered beam is used to update the wave-front corrector. We assume that changes in the atmosphere are on a slow time scale compared to the iterations of the feedback system, so

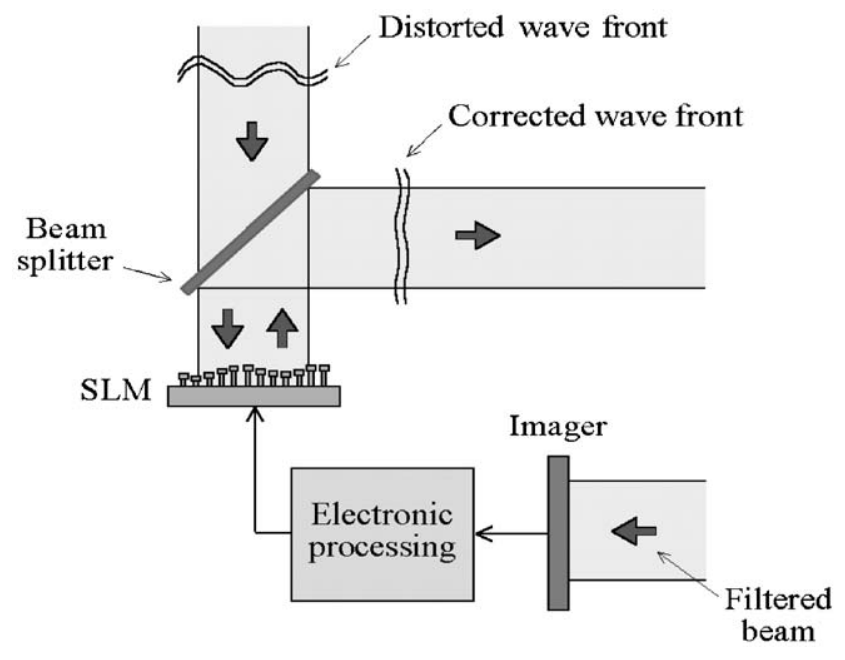

Fig. 2. Opto-electronically controlled wave-front corrector. An SLM modulates the distorted wave-front (ideally, cancelling the wave-front distortion). The SLM is updated based on an optical control beam (the filtered beam in Fig. 1).

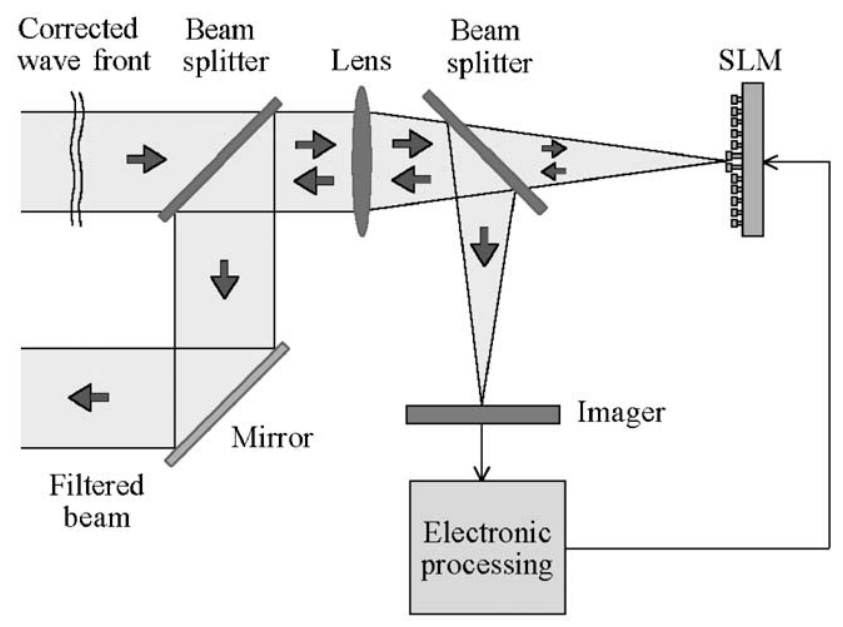

Fig. 3. Opto-electronically controlled spatial Fourier filter. The corrected beam in Fig. 1 is Fourier transformed by the lens, and the SLM modulates the Fourier-domain complex envelope. The SLM is controlled by the Fourier-domain intensity image, which is captured by the imager.

that for purposes of the analysis, the input beam wave-front distortion may be considered static. Through the action of the feedback system, the wave-front corrector SLM "learns" the (phase-conjugate) of the wave-front distortion present in the input beam.

A key feature of the feedback control system is that the spatial Fourier filter uses the same high-resolution SLM technology as the wave-front corrector, as shown in Fig. 3. As a result, the Fourier filter is a controlled Fourier filter, capable of changing with time as the feedback system evolves. The changes in the Fourier filter are not to respond to changes in the input beam distortion (which is assumed to be frozen), but are instead to overcome an intrinsic weakness of conventional phase-contrast techniques (as is further explained in Section 3.) 
The Fourier filters we consider operate only on the phase of the Fourier transform, leaving the magnitude of the Fourier transform unchanged. The control system design problem involves choosing the wave-front corrector update law and the Fourier filter update law so as to ensure stability and convergence to a wave-front-distortion-free corrected beam in as few iterations as possible.

Both the wave-front corrector and the controlled Fourier filter use a high-resolution imager in conjunction with the high-resolution SLM (with a one-to-one correspondence between imager pixels and SLM pixels), as shown in Figs. 2 and 3. What makes the control scheme practical is that simple parallel, distributed processing can be used between the imagers and SLMs in both the wave-front corrector (Fig. 2) and the Fourier filter (Fig. 3). That is, each SLM pixel is driven by the corresponding camera pixel (with any additional controls being common to all pixels). This feature, referred to in adaptive optics as "direct control," enables the resolution to scale without affecting system speed (Vorontsov et al., 2001; Justh et al., 2001; Vorontsov, Shmalhauzen, \& Koriabin, 1988; Vorontsov, Kirakosyan, \& Larichev, 1991; Pepper, Gaeta, \& Mitchell, 1995).

The system of Fig. 1 has its roots in the phase-contrast technique developed by Frits Zernike during the 1930s (for which he was later awarded a Nobel Prize in physics) (Zernike, 1955; Ferwerda, 1994; Goodman, 1996). Fig. 3 can be viewed as a modern incarnation of Zernike's idea (see also Fig. 6 in Appendix A, where a brief summary of the phase-contrast technique is given). Zernike's technique has found application in phase-contrast microscopes for many years (Pluta \& Szyjer, 1994). Recent advances in high-resolution SLMs have led to renewed interest in phase-contrast techniques for various applications, including adaptive optics (Vorontsov et al., 2001; Justh et al., 2001; Ivanov, Sivokon, \& Vorontsov, 1992; Glückstad, 1995).

The system of Fig. 1 is a nonlinear feedback system, because the filtered beam intensity image is a nonlinear functional of the corrected beam wave-front phase. The Fourier filter operator, i.e., the mapping from the Fourier-domain imager to the Fourier filter, introduces further nonlinearity, and this additional source of nonlinearity turns out to be essential to producing a practical wave-front control system. Addressing these nonlinear effects is the main contribution of the analysis presented here (and in Justh et al., 2000a; Justh \& Krishnaprasad, 2001).

\section{Mathematical models}

The key to successfully analyzing this type of system is to capture the underlying physics with sufficient fidelity, while keeping the nonlinear modeling simple enough to yield qualitative insights beyond what a linearized approximation to the dynamics can provide. To describe the coherent optical field (for a monochromatic beam), we introduce a complex envelope $A(x, y, z)$. We distinguish the $z$-direction as the "optical axis," and denote the transverse coordinates as $\mathbf{r}=$ $(x, y)$. The underlying electromagnetic field component that $A(\mathbf{r}, z)$ represents is then obtained by taking the real part of $A(\mathbf{r}, z) \mathrm{e}^{\mathrm{i}(\omega t-k z)}$, where $k=2 \pi / \lambda$ and $\omega=k c$ (with $\lambda$ the optical wavelength, and $c$ the speed of light).

The complex envelope $A(\mathbf{r}, z)$ can be expressed in polar form as

$A(\mathbf{r}, z)=a(\mathbf{r}, z) \mathrm{e}^{\mathrm{i} \phi(\mathbf{r}, z)}$,

where $[a(\mathbf{r}, z)]^{2}$ is proportional to the intensity, and $\phi(\mathbf{r}, z)$ is the phase (the quantity we are interested in measuring and controlling). The intensity is what a camera would measure if placed perpendicular to the optical axis in a plane containing the point $(0,0, z)$.

In the wave-front control setting, we are interested in how the phase in a particular plane $z=z_{0}$ along the optical axis evolves in time. Therefore, we drop the argument $z$ from Eq. (1), and we allow $A$ to depend on a time variable $t$. (This time variable corresponds to quasi-static changes in the complex envelope, not the time scale of electromagnetic field oscillations.) The feedback system evolves over time $t$; our assumption that the atmosphere is static implies that the distorted input beam is independent of $t$.

Our model of the feedback system shown in Fig. 1 uses the Fourier series representation:

$A(\mathbf{r}, t)=\sum_{\mathbf{p}} \mathrm{a}_{\mathbf{p}}(t) \mathrm{e}^{\mathrm{i}(2 \pi / \gamma) \mathbf{p} \cdot \mathbf{r}}$,

$\mathrm{a}_{\mathbf{p}}(t)=\frac{1}{\gamma^{2}} \int A(\mathbf{r}, t) \mathrm{e}^{-\mathrm{i}(2 \pi / \gamma) \mathbf{p} \cdot \mathbf{r}} \mathrm{d} \mathbf{r}$,

where $\mathbf{p}$ is an ordered pair of integers (i.e., $\mathbf{p}$ takes values in the integer lattice in the plane), and $\gamma$ is a parameter determining the Fourier-transform-domain resolution. For this representation to be valid, the complex envelope $A(\mathbf{r}, t)$ must be spatially periodic. In fact, we must have

$A\left(x+m_{x} \gamma, y+m_{y} \gamma, t\right)=A(x, y, t)$,

where $m_{x}$ and $m_{y}$ are arbitrary integers, and the integral in Eq. (2) is actually an integral over a square region $\Omega$ with sides of length $\gamma$. The validity of approximating beams in the physical system with spatially periodic functions is based on the assumption that for each beam in the physical system, its complex envelope is identically zero (for all time) outside of $\Omega$. The physically significant complex envelope is recovered from the spatially periodic representation simply by setting the complex envelope identically equal to zero outside of the region $\Omega$. For purposes of the analysis, however, it is convenient to work with the spatially periodic functions.

In this section we analyze three important cases, corresponding to three different choices of the Fourier filter in Fig. 3. Although in Fig. 3 the Fourier filter can evolve in time, driven by the imager and electronic processing, we only have precise mathematical results for models in which 
the Fourier filter is fixed for all time $t$. This analysis for fixed Fourier filters serves as a starting point for understanding (in an intuitive sense, backed up by experimental and simulation results (Justh et al., 2001; Justh, Vorontsov, Carhart, Beresnev, \& Krishnaprasad, 2000b)) the behavior of more general systems in which the Fourier filter changes with time (as described in Section 4).

\subsection{Single-pixel Fourier phase filter}

Remark on modeling assumptions: To simplify the analysis, we assume that time is continuous (although in practice, the system of Fig. 1 would be a discrete-time system), and we further use a continuum approximation to the wave-front-correcting phase distribution (which is motivated by the high-resolution SLM assumption). A discrete-time implementation of the system can be considered to be a forward-Euler method approximating the corresponding continuous-time system analyzed below. The continuum approximation for the high-resolution wave-front corrector simplifies the modeling, but is not essential. (In particular, Section 4.4 considers a spatially discrete model.)

Suppose only the zero-order Fourier component is phase-shifted by the SLM in Fig. 3 (which would correspond to the displacement of a single pixel located in the center of the SLM). Let the distorted beam complex envelope in Fig. 1 be represented as $a(\mathbf{r}) \mathrm{e}^{\mathrm{i} \phi(\mathbf{r})}$, and let the wave-front-correcting SLM impose a phase distribution $u(\mathbf{r}, t)$ on the distorted beam. The corrected beam is then represented by $a(\mathbf{r}) \mathrm{e}^{\mathrm{i}[u(\mathbf{r}, t)+\phi(\mathbf{r})]}$. We denote the filtered image by $[f(u+\phi)](\mathbf{r}, t)$ to emphasize that it is a functional of the phase of the corrected wave front. The evolution equation we assume for $u$ is

$\frac{\partial u}{\partial t}=\eta\left[l^{2} \Delta u-f(u+\phi)\right]$,

where the gain function $\eta$ satisfies $\eta(\mathbf{r}, t)>0, \forall \mathbf{r}, t$. The diffusion term is required for the analysis, but $l>0$ may be arbitrarily small.

The dynamics are thus determined by $f$, which captures the effects of the Fourier phase filtering of the corrected beam. Besides the phase-shift of the zero-order Fourier component, there is also an intensity measurement included in $f$. Letting $\theta$ represent the phase-shift of the zero-order Fourier component, we thus obtain the following model for the conventional Zernike wave-front sensor (see Appendix A):

$$
\begin{aligned}
& {\left[f_{\text {conv }}(u+\phi)\right](\mathbf{r}, t)} \\
& =\mid a(\mathbf{r}) \mathrm{e}^{\mathrm{i}[u(\mathbf{r}, t)+\phi(\mathbf{r})]}+\left(\mathrm{e}^{\mathrm{i} \theta}-1\right) \\
& \quad \times\left.\frac{1}{\gamma^{2}} \int a(\hat{\mathbf{r}}) \mathrm{e}^{\mathrm{i}[u(\hat{\mathbf{r}}, t)+\phi(\hat{\mathbf{r}})]} \mathrm{d} \hat{\mathbf{r}}\right|^{2} .
\end{aligned}
$$

(We have ignored finite aperture effects by failing to truncate the Fourier series at some finite frequency.)
Some of the undesirable nonlinearities present in $f_{\text {conv }}$ can be cancelled by taking the difference of two such images, corresponding to oppositely directed Fourier phase-shifts (Vorontsov et al., 2001; Justh et al., 2000a, b, 2001) (a related idea can be found in Seward, Lacombe, \& Giles, 1999). The resulting "differential" Zernike wave-front sensor model is

$$
\begin{aligned}
& {\left[f_{\text {diff }}\right.}u+\phi)](\mathbf{r}, t) \\
&=\mid a(\mathbf{r}) \mathrm{e}^{\mathrm{i}[u(\mathbf{r}, t)+\phi(\mathbf{r})]}+\left(\mathrm{e}^{\mathrm{i} \theta}-1\right) \\
& \quad \times\left.\frac{1}{\gamma^{2}} \int a(\hat{\mathbf{r}}) \mathrm{e}^{\mathrm{i}[u(\hat{\mathbf{r}}, t)+\phi(\hat{\mathbf{r}})]} \mathrm{d} \hat{\mathbf{r}}\right|^{2} \\
& \quad-\mid a(\mathbf{r}) \mathrm{e}^{\mathrm{i}[u(\mathbf{r}, t)+\phi(\mathbf{r})]}+\left(\mathrm{e}^{-\mathrm{i} \theta}-1\right) \\
& \quad \times\left.\frac{1}{\gamma^{2}} \int a(\hat{\mathbf{r}}) \mathrm{e}^{\mathrm{i}[u(\hat{\mathbf{r}}, t)+\phi(\hat{\mathbf{r}})]} \mathrm{d} \hat{\mathbf{r}}\right|^{2} \\
&=-4 \sin \theta \operatorname{Im}\left\{a(\mathbf{r}) \mathrm{e}^{-\mathrm{i}[u(\mathbf{r}, t)+\phi(\mathbf{r})]}\right. \\
&\left.\quad \times \frac{1}{\gamma^{2}} \int a(\hat{\mathbf{r}}) \mathrm{e}^{\mathrm{i}[u(\hat{\mathbf{r}}, t)+\phi(\hat{\mathbf{r}})]} \mathrm{d} \hat{\mathbf{r}}\right\} .
\end{aligned}
$$

The image subtraction required for the differential wave-front sensor can potentially be incorporated into the circuitry of the imager in Fig. 2 (Gruev \& Etienne-Cummings, 2000). The differential Zernike wave-front sensor image given by Eq. (6) is straightforward to interpret. At each point $\mathbf{r}$ (and for fixed $t$ ), the value of the operator $f_{\text {diff }}$ is a periodic function of the corrected beam phase. However, there is also global coupling through the zero-order Fourier component $1 / \gamma^{2} \int a(\mathbf{r}) \mathrm{e}^{\mathrm{i}[u(\mathbf{r}, t)+\phi(\mathbf{r})]} \mathrm{d} \mathbf{r}$. Also, Eq. (6) indicates that a judicious choice for $\theta$ is $\theta=\pi / 2$.

The dynamics given by Eq. (4), with $l=0$ and $f$ given by Eq. (6), are (formally) gradient dynamics with respect to the energy functional

$[V(u)](t)=-2 \gamma^{2} \sin \theta\left|\frac{1}{\gamma^{2}} \int a(\mathbf{r}) \mathrm{e}^{\mathrm{i}[u(\mathbf{r}, t)+\phi(\mathbf{r})]} \mathrm{dr}\right|^{2}$,

which is proportional to (the negative of) the intensity in the zero-order Fourier component of the corrected beam (Justh et al., 2001, 2000a).

Remark on notation: We will generally drop the $\mathbf{r}$ and $t$ arguments of $u$ and $f(u+\phi)$, as well as the $\mathbf{r}$ argument of $a$ and $\phi$, in the remainder of the development. The equations are then more compact and easy to read, but $a, \phi$, and $u$ must be interpreted as functions, and $f$ as an operator.

Using variational calculus, for Eq. (4) with $l=0$, we obtain

$$
\begin{aligned}
\frac{\mathrm{d} V}{\mathrm{~d} t} & =\frac{\delta V}{\delta u} \cdot \frac{\partial u}{\partial t} \\
& =4 \sin \theta \operatorname{Re}\left\{\int a(\hat{\mathbf{r}}) \mathrm{e}^{\mathrm{i}(u+\phi)} \mathrm{d} \hat{\mathbf{r}}\right.
\end{aligned}
$$




$$
\begin{aligned}
& \left.\times \frac{1}{\gamma^{2}} \int i a \mathrm{e}^{-\mathrm{i}(u+\phi)} \frac{\partial u}{\partial t} \mathrm{~d} \mathbf{r}\right\} \\
= & -\int\left(4 \operatorname { s i n } \theta \operatorname { I m } \left\{a \mathrm{e}^{-\mathrm{i}(u+\phi)}\right.\right. \\
& \left.\left.\times \frac{1}{\gamma^{2}} \int a \mathrm{e}^{\mathrm{i}(u+\phi)} \mathrm{d} \hat{\mathbf{r}}\right\}\right) \frac{\partial u}{\partial t} \mathrm{~d} \mathbf{r} \\
= & -\int \frac{1}{\eta}\left(\frac{\partial u}{\partial t}\right)^{2} \mathrm{~d} \mathbf{r},
\end{aligned}
$$

where $(\delta V / \delta u) \cdot v=\lim _{\varepsilon \rightarrow 0}[V(u+\varepsilon v)-V(u)] / \varepsilon$. Observe that $\mathrm{d} V / \mathrm{d} t \leqslant 0$, and $\mathrm{d} V / \mathrm{d} t=0$ only at equilibria of the $\mathrm{dy}-$ namics. The feedback system thus evolves to maximize the power in the zero-order Fourier component of the corrected beam. It is clear that $u(\mathbf{r}, t)=-\phi(\mathbf{r})$ minimizes $V(u)$, so that phase correction (or phase conjugation) corresponds to energy functional minimization. A standard performance metric for adaptive optic systems is the Strehl ratio, St, which is the normalized zero-order Fourier component intensity,

$[S t(u)](t)=\frac{\left|1 / \gamma^{2} \int a(\mathbf{r}) \mathrm{e}^{\mathrm{i}[u(\mathbf{r}, t)+\phi(\mathbf{r})]} \mathrm{d} \mathbf{r}\right|^{2}}{\left(1 / \gamma^{2} \int a(\mathbf{r}) \mathrm{d} \mathbf{r}\right)^{2}}$.

The Lyapunov functional for the single-pixel Fourier filter is thus proportional to the Strehl ratio (Justh et al., 2001, 2000a).

Strehl ratio maximization corresponds to wave-front correction, so from the forgoing analysis it would seem reasonable to conclude that the single-pixel Fourier filter is a good choice for the Fourier filter in Fig. 3. Indeed, when the Strehl ratio of the corrected beam in Fig. 1 is sufficiently high, the single-pixel Fourier filter is an excellent choice. The practical difficulty occurs when detector noise is present and the Strehl ratio of the corrected beam is low. When the Strehl ratio is low, the wave-front sensor image $f_{\text {diff }}$ given by Eq. (6) has low contrast (which, in the presence of detector noise, implies a low signal-to-noise ratio). For this reason, we are led to consider alternative choices for the Fourier filter that can provide improved contrast.

\subsection{General Fourier phase filter with common phase shift}

If a common phase shift $\theta$ is applied to multiple Fourier components, then the (differential) wave-front sensor image (in the absence of any correction) becomes

$$
\begin{aligned}
& {\left[f_{\text {common }}(\phi)\right](\mathbf{r})} \\
& =\mid a \mathrm{e}^{\mathrm{i} \phi}+\left(\mathrm{e}^{\mathrm{i} \theta}-1\right) \sum_{\mathbf{p} \in I}\left(\frac{1}{\gamma^{2}} \int a \mathrm{e}^{\mathrm{i} \phi} \mathrm{e}^{-\mathrm{i}(2 \pi / \gamma) \mathbf{p} \cdot \hat{\mathbf{r}}} \mathrm{d} \hat{\mathbf{r}}\right) \\
& \\
& \quad \times\left.\mathrm{e}^{\mathrm{i}(2 \pi / \gamma) \mathbf{p} \cdot \mathbf{r}}\right|^{2}
\end{aligned}
$$

$$
\begin{aligned}
& -\mid a \mathrm{e}^{\mathrm{i} \phi}+\left(\mathrm{e}^{-\mathrm{i} \theta}-1\right) \sum_{\mathbf{p} \in I}\left(\frac{1}{\gamma^{2}} \int a \mathrm{e}^{\mathrm{i} \phi} \mathrm{e}^{-\mathrm{i}(2 \pi / \gamma) \mathbf{p} \cdot \hat{\mathbf{r}}} \mathrm{d} \hat{\mathbf{r}}\right) \\
& \times\left.\mathrm{e}^{\mathrm{i}(2 \pi / \gamma) \mathbf{p} \cdot \mathbf{r}}\right|^{2} \\
& =-4 \sin \theta \sum_{\mathbf{p} \in I} \operatorname{Im}\left\{a \mathrm{e}^{-\mathrm{i}(\phi-(2 \pi / \gamma) \mathbf{p} \cdot \mathbf{r})}\right. \\
& \left.\times \frac{1}{\gamma^{2}} \int a \mathrm{e}^{\mathrm{i}(\phi-(2 \pi / \gamma) \mathbf{p} \cdot \hat{\mathbf{r}})} \mathrm{d} \hat{\mathbf{r}}\right\}
\end{aligned}
$$

where $I$ is a finite index set that may or may not contain $\mathbf{0}$ (the zero-order component). We assume for purposes of the results presented below that both $I$ and $\theta$ are fixed for all time $t$.

To state rigorous results for the system of Fig. 1, we make the following hypotheses:

(A.1) The boundary conditions are periodic on $\Omega$, a square region with sides of length $\gamma$ (and all integrals are understood to be integrals over $\Omega$ ).

(A.2) The initial conditions satisfy $u(\mathbf{r}, 0), D u(\mathbf{r}, 0), \phi(\mathbf{r})$, $D \phi(\mathbf{r}) \in L^{2}(\Omega)$.

(A.3) $\eta_{\min }<\eta(\mathbf{r})<\eta_{\max }$ on $\Omega$, where $\eta_{\min }$ and $\eta_{\max }$ are positive constants.

(A.4) $0<\theta<\pi$.

(A.5) $l>0$.

(A.6) $I$ is a finite set.

(A.7) $\int[a(\mathbf{r})]^{2} \mathrm{~d} \mathbf{r}$ is bounded.

Proposition 1. Under assumptions (A.1)-(A.7), weak solutions $u(\mathbf{r}, t)$ for Eq. (4), with $f$ given by Eq. (10), exist and are unique.

Proof. See Appendix B.

Proposition 2 (Justh et al., 2000a). Under assumptions (A.1)-(A.7), system (4), with $f(\phi)$ given by Eq. (10), is a gradient system with respect to the energy functional

$$
\begin{aligned}
V= & \int \frac{l^{2}}{2}|\nabla u|^{2} \mathrm{~d} \mathbf{r}-2 \gamma^{2} \\
& \times \sin \theta \sum_{\mathbf{p} \in I}\left|\frac{1}{\gamma^{2}} \int a(\mathbf{r}) \mathrm{e}^{\mathrm{i}(u(\mathbf{r}, t)+\phi(\mathbf{r})-(2 \pi / \gamma) \mathbf{p} \cdot \mathbf{r})} \mathrm{d} \mathbf{r}\right|^{2} .
\end{aligned}
$$

Specifically, $\partial u / \partial t=-\nabla_{u} V$ (with respect to the inner product $\langle g, h\rangle=\int(1 / \eta(\mathbf{r})) g(\mathbf{r}) h(\mathbf{r}) \mathrm{d} \mathbf{r}$ on $\left.L^{2}(\Omega)\right)$, and

$\frac{\mathrm{d} V}{\mathrm{~d} t}=-\int \frac{1}{\eta}\left(\frac{\partial u}{\partial t}\right)^{2} \mathrm{~d} \mathbf{r}$.

Thus, $V$ also serves as a Lyapunov functional for the $d y$ namics; i.e., $\mathrm{d} V / \mathrm{d} t \leqslant 0$, with $\mathrm{d} V / \mathrm{d} t=0$ only at equilibria. 
Proof. Differentiating the energy functional $V$ with respect to time gives

$$
\frac{\mathrm{d} V}{\mathrm{~d} t}=\frac{\delta V}{\delta u} \frac{\partial u}{\partial t}=-\int \frac{1}{\eta}\left(\frac{\partial u}{\partial t}\right)^{2} \mathrm{~d} \mathbf{r} .
$$

This calculation shows that $\partial u / \partial t=-\nabla_{u} V$, and that $\mathrm{d} V / \mathrm{d} t \leqslant 0$, with $\mathrm{d} V / \mathrm{d} t=0$ only at equilibria.

Remark. The term $\int l^{2} / 2|\nabla u|^{2}$ dr in Eq. (11) can be viewed as a regularization term, penalizing rapid changes in the $u(\mathbf{r}, t)$ with respect to the spatial variable $\mathbf{r}$. The second term of Eq. (11) is proportional to the sum of the intensities of the Fourier components of the corrected beam that are phase-shifted by the Fourier filter.

As time passes, the system thus tends to maximize the total intensity within the collection of phase-shifted pixels (provided $l$ is sufficiently small). The Strehl ratio (Eq. (9)) is not necessarily maximized when $I \neq\{\boldsymbol{0}\}$. However, if $I$ is chosen judiciously (and $\theta=\pi / 2$ ), $f_{\text {common }}$ given by Eq. (10) can provide a much higher signal-to-noise ratio (SNR) in the presence of detector noise than $f_{\text {diff }}$ given by Eq. (6). This motivates why we consider a time-varying Fourier filter in Fig. 3: we would like the index set $I$ to change as time passes so as to maintain a sufficiently high SNR, but to eventually converge to $I=\{\boldsymbol{0}\}$ so that the Strehl ratio is maximized. (For further discussion, see Section 4 below.)

\subsection{General Fourier phase filter with arbitrary phase shifts}

For practical reasons, we want to consider Fourier filters in which both the finite index set $I$, and the phase shifts $\theta_{\mathbf{p}}$ for each $\mathbf{p} \in I$, can be arbitrarily chosen. The (differential) wave-front sensor image for fixed, arbitrary Fourier component phase shifts is given by

$$
\begin{aligned}
f_{\operatorname{arb}}(\phi) & \mid a \mathrm{e}^{\mathrm{i} \phi}+\sum_{\mathbf{p} \in I}\left(\mathrm{e}^{\mathrm{i} \theta}-1\right)\left(\frac{1}{\gamma^{2}} \int a \mathrm{e}^{\mathrm{i} \phi} \mathrm{e}^{-\mathrm{i}(2 \pi / \gamma) \mathbf{p} \cdot \hat{\mathbf{r}}} \mathrm{d} \hat{\mathbf{r}}\right) \\
& \times\left.\mathrm{e}^{\mathrm{i}(2 \pi / \gamma) \mathbf{p} \cdot \mathbf{r}}\right|^{2} \\
& -\mid a \mathrm{e}^{\mathrm{i} \phi}+\sum_{\mathbf{p} \in I}\left(\mathrm{e}^{-\mathrm{i} \theta_{\mathbf{p}}}-1\right)\left(\frac{1}{\gamma^{2}} \int a \mathrm{e}^{\mathrm{i} \phi} \mathrm{e}^{-\mathrm{i}(2 \pi / \gamma) \mathbf{p} \cdot \hat{\mathbf{r}}} \mathrm{d} \hat{\mathbf{r}}\right) \\
& \times\left.\mathrm{e}^{\mathrm{i}(2 \pi / \gamma) \mathbf{p} \cdot \mathbf{r}}\right|^{2}
\end{aligned}
$$

$$
\begin{aligned}
= & -4 \sum_{\mathbf{p} \in I} \sin \theta_{\mathbf{p}} \operatorname{Im}\left\{a \mathrm{e}^{-\mathrm{i}(\phi-(2 \pi / \gamma) \mathbf{p} \cdot \mathbf{r})}\right. \\
& \left.\times \frac{1}{\gamma^{2}} \int a \mathrm{e}^{\mathrm{i}(\phi-(2 \pi / \gamma) \mathbf{p} \cdot \hat{\mathbf{r}})} \mathrm{d} \hat{\mathbf{r}}\right\}+f_{\mathrm{o}}(\phi),
\end{aligned}
$$

where the operator $f_{\mathrm{o}}(\phi)$ is given by

$$
\begin{aligned}
f_{\mathrm{o}}(\phi) & \mid \sum_{\mathbf{p} \in I}\left(\mathrm{e}^{\mathrm{i} \theta_{\mathbf{p}}}-1\right)\left(\frac{1}{\gamma^{2}} \int a \mathrm{e}^{\mathrm{i} \phi} \mathrm{e}^{-\mathrm{i}(2 \pi / \gamma) \mathbf{p} \cdot \hat{\mathbf{r}}} \mathrm{d} \hat{\mathbf{r}}\right) \\
& \times\left.\mathrm{e}^{\mathrm{i}(2 \pi / \gamma) \mathbf{p} \cdot \mathbf{r}}\right|^{2} \\
& -\mid \sum_{\mathbf{p} \in I}\left(\mathrm{e}^{-\mathrm{i} \theta_{\mathbf{p}}}-1\right)\left(\frac{1}{\gamma^{2}} \int a \mathrm{e}^{\mathrm{i} \phi} \mathrm{e}^{-\mathrm{i}(2 \pi / \gamma) \mathbf{p} \cdot \hat{\mathbf{r}}} \mathrm{d} \hat{\mathbf{r}}\right) \\
& \times\left.\mathrm{e}^{\mathrm{i}(2 \pi / \gamma) \mathbf{p} \cdot \mathbf{r}}\right|^{2} .
\end{aligned}
$$

By taking $f_{\mathrm{o}}(\phi) \equiv 0$ in Eq. (14), we obtain a nonlinear approximation to the wave-front sensor image. Simulation and experimental work suggest that this nonlinear approximation adequately captures the system's qualitative behavior (Justh et al., 2001).

Proposition 3 (Justh \& Krishnaprasad, 2001). Under assumptions (A.1)-(A.7), system (4), with $f=f_{\text {arb }}-f_{\mathrm{o}}$, is a gradient system with respect to the energy functional

$$
\begin{aligned}
V= & \int \frac{l^{2}}{2}|\nabla u|^{2} \mathrm{~d} \mathbf{r}-2 \gamma^{2} \\
& \times \sum_{\mathbf{p} \in I} \sin \theta_{\mathbf{p}}\left|\frac{1}{\gamma^{2}} \int a(\mathbf{r}) \mathrm{e}^{\mathrm{i}(u(\mathbf{r}, t)+\phi(\mathbf{r})-(2 \pi / \gamma) \mathbf{p} \cdot \mathbf{r})} \mathrm{d} \mathbf{r}\right|^{2} .
\end{aligned}
$$

Also, $V$ serves as a Lyapunov functional for the dynamics.

Proof. Analogous to the proof of Proposition 2.

\section{Practical implementation issues}

The analysis presented in Section 3 above, and in particular, Propositions 2 and 3, apply to particular choices of fixed (i.e., time-independent) Fourier filters. The single-pixel Fourier filter leads to Strehl ratio maximization (i.e., wave-front correction), but only in the noiseless setting. In the presence of noise, the single-pixel Fourier filter can suffer from low contrast (and low SNR) if the Strehl ratio is low. The fixed multi-pixel Fourier filter (with a common phase shift $\theta$ ) can produce higher-contrast images, and a convergent feedback system (by Proposition 2), but will 
not in general lead to wave-front correction (i.e., Strehl ratio maximization). The fixed multi-pixel Fourier filter with arbitrary phase shifts $\left(\theta_{\mathbf{p}}\right.$ for each $\left.\mathbf{p} \in I\right)$ can also produce higher-contrast images than the single-pixel Fourier filter, but the convergence result, Proposition 3, only applies to an approximation of the physics-based model (i.e., $f=f_{\text {arb }}-f_{\mathrm{o}}$ instead of $f=f_{\text {arb }}$ ).

In this section, we discuss how Propositions 2 and 3, along with the Lyapunov functionals given by Eqs. (7), (11), and (16), provide insight into how to implement a practical high-resolution wave-front control system. Specifically, the key aspect of a practical wave-front control system which remains to be specified is the time-evolution of the Fourier filter. The idea of using a time-varying Fourier filter is motivated by the above-mentioned strengths and weaknesses of the different fixed Fourier filters analyzed in the previous section. We discuss below a judicious choice for this time dependence. However, due to the complexity of the wave-front control system with a time-varying Fourier filter, here we depart from a rigorous analysis and take a more pragmatic approach, guided by the modeling and theory presented in the previous sections.

\subsection{Proportional Fourier filter operator}

There are different approaches one might take for prescribing the Fourier filter as a function of time, but we will focus here on one particular approach which has been demonstrated in simulation and experiments to perform well in practice. For concreteness, we will assume that the index set $I$ is fixed (i.e., time-independent). What can vary with time are the $\theta_{\mathbf{p}}$, for $\mathbf{p} \in I$. (The only constraint on $I$ is that it be a finite set; we may therefore choose $I$ so that it includes every addressable pixel of the Fourier filter SLM.) One choice for determining the $\theta_{\mathbf{p}}(t)$ is

$\theta_{\mathbf{p}}(t)=\alpha(t)\left|\mathrm{a}_{\mathbf{p}}(t)\right|^{2}$,

where $\left|\mathrm{a}_{\mathbf{p}}(t)\right|^{2}$ is proportional to the intensity of the pth Fourier series component of the corrected beam, and $\alpha(t)>0$ is a gain factor (which is time-varying, but p-independent) (Vorontsov et al., 2001; Ivanov et al., 1992). One possible choice for $\alpha(t)$ is

$\alpha(t)=\frac{\pi / 2}{\max _{\mathbf{p} \in I}\left|\mathrm{a}_{\mathbf{p}}(t)\right|^{2}}$,

so that $\max _{\mathbf{p} \in I} \theta_{\mathbf{p}}(t)=\pi / 2$ for each $t$. Because $\theta_{p}(t)$ is proportional to the intensity of the pth Fourier component, we refer to this approach as the "proportional Fourier filter operator" (Vorontsov et al., 2001; Ivanov et al., 1992).

To understand intuitively how the proportional Fourier filter operator works, consider $V$ (for a fixed Fourier filter with arbitrary phase shifts $\theta_{\mathbf{p}}$ ) given by Eq. (16), which can be rewritten as

$V=\int \frac{l^{2}}{2}|\nabla u|^{2} \mathrm{~d} \mathbf{r}-2 \gamma^{2} \sum_{\mathbf{p} \in I} \sin \theta_{\mathbf{p}}\left|\mathrm{a}_{\mathbf{p}}(t)\right|^{2}$.
For $l \ll \gamma$, so that we may neglect the $\int l^{2} / 2|\nabla u|^{2} \mathrm{~d} \mathbf{r}$ term, we see that $V$ is minimized by maximizing $\left|\mathrm{a}_{\hat{\mathbf{p}}}\right|^{2}$, where $\hat{\mathbf{p}}=\arg \max _{\mathbf{p} \in I} \theta_{\mathbf{p}}$ (where we assume that $0 \leqslant \theta_{\mathbf{p}} \leqslant \pi / 2$, $\forall \mathbf{p} \in I)$. Since the total beam intensity is finite, this suggests (due to Proposition 3) that there is a tendency for the $\hat{\mathbf{p}} \mathbf{t h}$ Fourier component of the corrected beam to grow at the expense of the other Fourier components. If instead of keeping the $\theta_{\mathbf{p}}$ fixed with time, we allow the $\theta_{\mathbf{p}}$ to vary according to Eqs. (17) and (18), then the growth of the pth Fourier component is reinforced by the growth of $\theta_{\hat{\mathbf{p}}}$ relative to the other $\theta_{\mathbf{p}}$.

The real utility of the proportional Fourier filter operator lies in its behavior when the Strehl ratio is initially very low, because unlike the single-pixel Fourier filter, the Fourier filter given by Eqs. (17) and (18) can still have adequate contrast (or SNR). The tendency of the system to maximize the intensity of Fourier components with larger phase shifts $\theta_{\mathbf{p}}$, reinforced by the rule that $\theta_{\mathbf{p}}$ is proportional at each time $t$ to the pth Fourier component intensity, results in a self-organizing, or winner-take-all behavior in the system. As a result of this winner-take-all behavior, the Fourier filter tends toward a single-pixel Fourier filter, although there is nothing to suggest that the "winning" single pixel will in fact be the zero-order pixel. If the "winning" pixel is not the zero-order pixel, then an additional "tilt" component is introduced in the corrected wave front. This tilt component can either be removed optically (using an active tip-tilt mirror system), or can be avoided by augmenting Eq. (17) (which is beyond the scope of this paper).

The proportional Fourier filter operator thus has all of the characteristics we require for a practical high-resolution wave-front control system. With the help of Propositions 2 and 3 we are able to develop an intuitive understanding of the behavior of the wave-front control system with the proportional Fourier filter. This is important because it appears to be quite difficult to prove a convergence result for the dynamics (4), with $f(\phi)$ given by Eq. (14) and $\theta_{\mathbf{p}}$ given by Eqs. (17) and (18).

\subsection{Interpretation of experimental results}

Recent experimental results clearly illustrate the Fourier filter evolution arising from the proportional Fourier filter operator (Justh, Vorontsov, Carhart, Beresnev, \& Krishnaprasad, 2000b). The experimental system consisted of the system of Fig. 1, but with a liquid-crystal light valve (LCLV) used as an optically controlled Fourier phase filter (Justh et al., 2001). The LCLV does not require the imager and electronic processing shown in Fig. 3, because the LCLV is designed to produce a phase shift distribution proportional to the intensity distribution incident upon it, as in Eq. (17).

The differential wave-front sensor approach with image subtraction was not used in these experiments, and this was the primary way in which the experimental system 

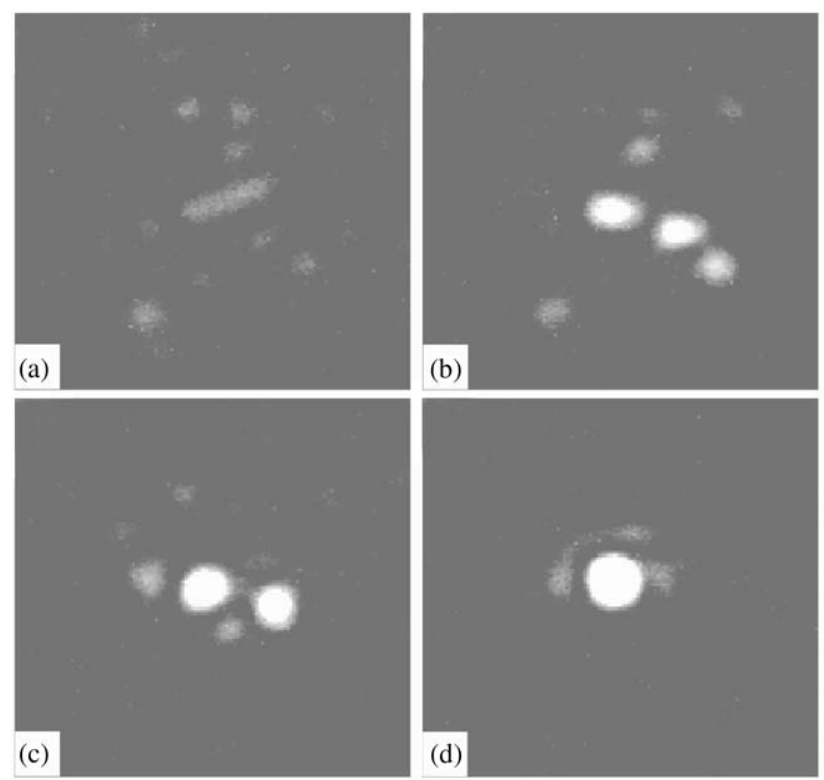

Fig. 4. Experimental results for LCLV-based feedback system: Fourier-domain intensity distribution (a) initially, (b) after 10 iterations, (c) after 20 iterations, and (d) after 30 iterations (from Justh et al., 2000b).

deviates from the models presented in this paper. Nevertheless, there was good qualitative agreement between the models and the experimental system over a certain range of operating conditions (for experimental details, see Justh et al., 2000b).

Fig. 4 shows the Fourier filter evolution for one experimental trial of the wave-front control system with the LCLV. Initially, because the wave front is highly distorted, the Fourier-domain intensity is distributed among many spatial frequency components. As the system evolves, the Fourier-domain intensity becomes concentrated in several components, then in only two components, and ultimately in a single component (Justh et al., 2000b).

\subsection{Intensity scintillations}

From a practical standpoint, one of the key features of Propositions 1, 2, and 3 is that the input beam intensity can have spatial variation, which is embodied in the dependence of the amplitude $a$ on $\mathbf{r}$. Laser beams which pass through long, horizontal atmospheric paths are typically subject to phase distortion, followed by diffraction, followed by further phase distortion, and so on, as the beam passes through successive regions of turbulence (Roggemann \& Welsh, 1996). Diffraction transforms phase distortion into both intensity and phase distortion, so that the beam emerging from the long atmospheric path through turbulence has intensity scintillations in addition to phase distortion. The ability of the high-resolution wave-front control system of Fig. 1 (together with differential Zernike wave-front sensing, as in Eq. (6)) to perform wave-front correction in the presence of input beam intensity variations is thus of considerable importance for applications involving laser beam propagation through long atmospheric paths.

\subsection{Low-resolution wave-front correction with high-resolution sensing}

The system of Fig. 1 is an alternative to conventional low-resolution adaptive optics systems based on wave-front slope measurements (Roggemann \& Welsh, 1996; Hardy, 1978; Roddier, 1999). The devices generally used for conventional adaptive-optic wave-front correction have resolutions on the order of 10-500 elements. Piezoelectrically actuated deformable mirrors are an example of this type of low-resolution adaptive optic wave-front corrector, and advantages of deformable mirrors include large deformations (i.e., several wavelengths of stroke), nearly 100 percent fill factor, and commercial availability. Depending on the extent of wave-front aberrations, low-resolution wave-front correction can be quite adequate for atmospheric turbulence compensation in many cases.

In conventional adaptive optics systems with lowresolution wave-front correctors, low-resolution ShackHartmann wave-front sensing is typically used. In such systems, the deformable mirror control signals are derived from the (Shack-Hartmann sensor) wave-front slope measurements, taking into account the deformable mirror influence function (i.e., the function describing the dependence of the continuous mirror displacement distribution on the discrete actuator control signals). The assumption that the input beam wave front can be reconstructed only from measurements of its gradient introduces errors when the wave-front distortion contains "branch points" (Fried, 1998). A branch point is a singularity in the phase distribution such that summing up phase differences around such a point produces an integer multiple of $2 \pi$ (instead of zero). Highly aberrated beams, such as those which traverse long horizontal paths through atmospheric turbulence, tend to contain many such branch points.

Because the Zernike phase-contrast wave-front sensor measures wave front directly, and does not involve reconstruction of the wave front from gradient measurements, branch points cause no difficulties for the system of Fig. 1. It is therefore natural to consider the advanced phase-contrast wave-front sensor as a wave-front sensor for a low-resolution adaptive optic system, and compare its performance to conventional low-resolution adaptive optic systems (although a detailed comparison of this type is beyond the scope of this paper.).

The first step is to properly spatially discretize the dynamics so that convergence results analogous to Propositions 2 and 3 carry over to the discretized system. Let the wavefront correction element be described by

$u(\mathbf{r}, t)=S\left(\mathbf{r}, u_{11}(t), u_{12}(t), \ldots, u_{n n}(t)\right)$,

where the $u_{i j}(t)$ are electrode voltages causing deformation of, say, a deformable mirror. A natural choice for a 
discrete approximation to Eq. (4), corresponding to principal component analysis, is

$$
\begin{aligned}
\frac{\mathrm{d} u_{i j}}{\mathrm{~d} t}= & l^{2}\left(\frac{u_{(i-1) j}+u_{i(j-1)}+u_{(i+1) j}+u_{i(j+1)}-4 u_{i j}}{\delta^{2}}\right) \\
& -\int \frac{\partial S}{\partial u_{i j}} f\left(S\left(\mathbf{r}, u_{11}, u_{12}, \ldots, u_{n n}\right)+\phi\right) \mathrm{d} \mathbf{r} .
\end{aligned}
$$

The parameter $\delta$ corresponds to the length scale of the spatial discretization of the wave-front corrector. (For a practical implementation, standard practice would be to approximate the $\partial S / \partial u_{i j}$ as functions of $\mathbf{r}$ alone.)

From expression (21) for the dynamics, we see that it is not necessary to measure $f(u+\phi)$, only certain spatially weighted functionals of $f(u+\phi)$. Thus, as long as the response of an array of photodetectors is appropriately matched to a finite degree-of-freedom wavefront corrector, phase distortion suppression may be achievable. Furthermore, the photodetector signals could be directly fed back to the corresponding electrodes of the phase-correcting SLM, with the only computation required being the subtraction needed for the differential approach (and integration with respect to time).

Under appropriate hypotheses, it can be shown (see Justh et al., 2000a) that the dynamical system (21) is a gradient system with respect to the energy function $V_{\delta}$ given by

$$
\begin{aligned}
V_{\delta}= & \frac{l^{2}}{\delta^{2}} \sum_{i=1}^{n} \sum_{j=1}^{n}\left(2\left(u_{i j}\right)^{2}-u_{(i-1) j} u_{i j}-u_{i(j-1)} u_{i j}\right)-2 \gamma^{2} \\
& \times \sin \theta \sum_{\mathbf{p} \in I}\left|\frac{1}{\gamma^{2}} \int a(\mathbf{r}) \mathrm{e}^{\mathrm{i}\left(S\left(\mathbf{r}, u_{11}, \ldots, u_{n n}\right)+\phi-(2 \pi / \gamma) \mathbf{p} \cdot \mathbf{r}\right)} \mathrm{d} \mathbf{r}\right|^{2} .
\end{aligned}
$$

Specifically, $\mathrm{d} u_{i j} / \mathrm{d} t=-\partial V_{\delta} / \partial u_{i j}$, and

$$
\frac{\mathrm{d} V_{\delta}}{\mathrm{d} t}=-\sum_{i=1}^{n} \sum_{j=1}^{n}\left(\frac{\mathrm{d} u_{i j}}{\mathrm{~d} t}\right)^{2} .
$$

Thus, $V_{\delta}$ also serves as a Lyapunov function for the dynamics; i.e., $\mathrm{d} V_{\delta} / \mathrm{d} t \leqslant 0$, with $\mathrm{d} V_{\delta} / \mathrm{d} t=0$ only at equilibria. Observe that for $l=0$ and $I=\{\boldsymbol{0}\}$, minimizing $V_{\delta}$ corresponds to maximizing the Strehl ratio.

A practical drawback for the scheme we have just described, using phase-contrast sensing with low-resolution wave-front correction, is that phase distortion greater than a wavelength may not be correctly compensated because the wave-front sensor does not distinguish between multiples of $2 \pi$ in phase. However, by combining a conventional low-resolution adaptive optic system with a high-resolution adaptive optic system (or perhaps simply a high-resolution phase-contrast wave-front sensor based on the controlled Fourier filter of Fig. 3), the effects of branch points could potentially be greatly mitigated.

\section{Wave-front estimation}

In the preceding sections, we considered deterministic models and used methods from nonlinear dynamics to study stability and convergence for certain high-resolution wave-front control systems. We also considered various practical issues, including implementability, and wave-front sensor image contrast when the Strehl ratio is low. While the deterministic analysis provides considerable guidance for designing a high-resolution wave-front control system, the one key piece of information it cannot tell us is how to choose the feedback gain function $\eta(\mathbf{r}, t)$. For choosing $\eta(\mathbf{r}, t)$, it is necessary to consider the statistical nature of atmospheric turbulence and photodetector noise.

In this section we formulate a wave-front estimation problem which parallels the (deterministic) wave-front control problem addressed in the preceding sections. Specifically, we discretize both the time and spatial variables, and we consider the system of Fig. 1 to be a nonlinear estimator: based on noisy, nonlinear measurements of the corrected beam wave front, the system attempts to estimate the (conjugate of the) input beam wave front.

\subsection{Modeling wave-front distortion and photon noise}

Very detailed statistical models of atmospheric turbulence and photon noise can be found in the literature, and have been used to study and design adaptive optic systems (Roggemann \& Welsh, 1996). Statistical models for atmospheric turbulence generally assume particular forms for the spatial power spectra, with origins in the study of thermal energy transfer and fluid motion across various length scales. Motion of the turbulent structures produces the temporal dependence of the wave-front distortion.

A standard approach for modeling photon noise is the semi-classical model (Roggemann \& Welsh, 1996). Electromagnetic field theory is used to describe the system up to the plane where the intensity measurement is made. The intensity measurement is then taken to be a Poisson process with its rate function determined by the classical electromagnetic field irradiance. (Integrating the rate function over the area of a single photodetector gives the average number of photons incident on the detector per unit time.) The electromagnetic field irradiance depends on both the deterministic optical system (i.e., the operator $f$ of Section 3), and the random atmospheric turbulence.

\subsection{Nonlinear estimator}

We let $k$ denote the discrete time variable, and we let $\mathbf{s}$, a two-dimensional vector of integers, denote the discrete transverse coordinates. For the wave-front estimation 
problem we use a discrete Fourier transform:

$A_{\mathbf{s}}=\sum_{\mathbf{p}} \mathrm{a}_{\mathbf{p}} \mathrm{e}^{\mathrm{i}(2 \pi / n) \mathbf{p} \cdot \mathbf{s}}$

$\mathrm{a}_{\mathbf{p}}=\frac{1}{n^{2}} \sum_{\mathbf{s}} A_{\mathbf{s}} \mathrm{e}^{-\mathrm{i}(2 \pi / n) \mathbf{p} \cdot \mathbf{s}}$,

where the $a_{p}$ now represent discrete Fourier transform coefficients. The total number of grid points (in both the spatial and Fourier domains) is $n^{2}$.

The state of the distortion (specifically, the effect of the atmospheric turbulence on input beam phase) is denoted $\phi_{\mathbf{s}}(k)$. We assume that the update equation for the atmosphere is

$\phi_{\mathbf{s}}(k+1)=\phi_{\mathbf{s}}(k)+w_{\mathbf{s}}(k)$,

where $w_{\mathbf{s}}(k)$ is a noise process (assumed to be stationary) driving the state of the atmospheric distortion.

The estimate of the state of the distortion at time $k$ (given information up to time $k-1)$ is denoted $\hat{\phi}_{\mathbf{s}}(k \mid k-1)$. The error signal,

$\varepsilon_{\mathbf{s}}(k)=\phi_{\mathbf{s}}(k)-\hat{\phi}_{\mathbf{s}}(k \mid k-1)$,

is produced by the phase-correcting SLM, and is what we would like the wave-front control system to minimize (in an appropriately weighted mean-square sense). We let $\varepsilon(k)$ denote the matrix $\left\{\varepsilon_{\mathbf{s}}(k)\right\}$. In the absence of measurement noise, the image measured by, e.g., the single-pixel differential Zernike wave-front sensor can then be expressed as

$[f(\varepsilon)]_{\mathbf{s}}(k)=-4 \sin \theta \operatorname{Im}\left\{a_{\mathbf{s}} \mathrm{e}^{-\mathrm{i} \varepsilon_{\mathbf{s}}(k)} \frac{1}{n^{2}} \sum_{\overline{\mathbf{s}}} a_{\overline{\mathbf{s}}} \mathrm{e}^{\mathrm{i} \varepsilon_{\bar{s}}(k)}\right\}$.

The measured image, including a photon noise term $v_{\mathbf{s}}(k)$ (assumed to be stationary), is then $[f(\varepsilon)]_{\mathbf{s}}(k)+v_{\mathbf{s}}(k)$.

A natural update equation for the estimator is

$\hat{\phi}_{\mathbf{s}}(k+1 \mid k)=\hat{\phi}_{\mathbf{s}}(k \mid k-1)+c_{\mathbf{s}}(k)\left([f(\varepsilon)]_{\mathbf{s}}(k)+v_{\mathbf{s}}(k)\right)$,

where $\left\{c_{\mathbf{s}}(k)\right\}$ is a gain matrix (replacing the gain function $\eta(\mathbf{r}, t)$ of Section 3$)$. The block diagram corresponding to this estimator is shown in Fig. 5. Eq. (28) is a discrete-time (and spatially discretized) version of the deterministic gradient flow (4), with $l=0$, and with additional noise terms. The evolution equation for the error is

$\varepsilon_{\mathbf{S}}(k+1)=\varepsilon_{\mathbf{S}}(k)-c_{\mathbf{s}}(k)\left([f(\boldsymbol{\varepsilon})]_{\mathbf{s}}(k)+v_{\mathbf{s}}(k)\right)+w_{\mathbf{s}}(k)$.

We thus have a nonlinear update equation for the estimation error, involving both process and measurement noise.

The "ODE method" relates the stability of the stochastic system (28) to a system of deterministic ordinary differential equations (Kushner \& Yin, 1997; Ljung \& Soderstrom, 1983). This system of deterministic ordinary differential equations corresponds to a discretization of the PDE model (4) for $l=0$. We are currently investigating the implications of the ODE method for system (28).

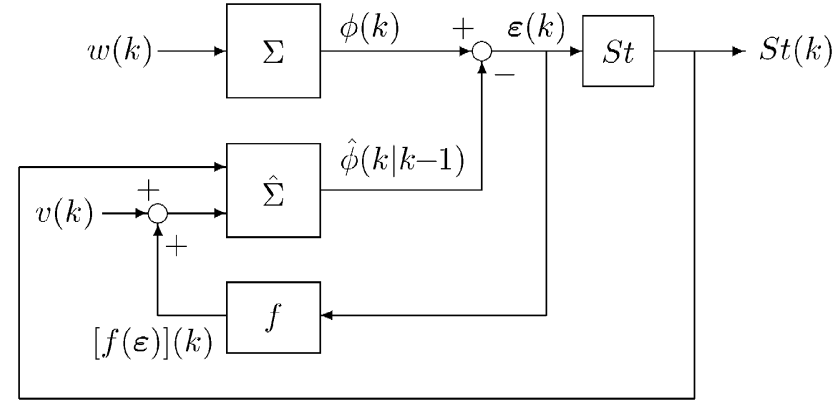

Fig. 5. Wave-front estimator block diagram. Observe that the error process (rather than the output process) is fed back through the nonlinearity.

\subsection{Relationship between Strehl ratio and error covariance}

A natural performance metric for wave-front estimation is the instantaneous (weighted) sample error covariance (over the spatial extent of the beam cross-section at the wave-front corrector)

$\varphi(\varepsilon)=\frac{1 / n^{2} \sum_{\mathbf{s}} a_{\mathbf{s}}\left(\varepsilon_{\mathbf{s}}-\bar{\varepsilon}\right)^{2}}{1 / n^{2} \sum_{\mathbf{s}} a_{\mathbf{s}}}$,

where

$\bar{\varepsilon}=\frac{1 / n^{2} \sum_{\mathbf{s}} a_{\mathbf{s}} \varepsilon_{\mathbf{s}}}{1 / n^{2} \sum_{\mathbf{s}} a_{\mathbf{s}}}$.

The relationship between the Strehl ratio and the wave-front error covariance is well-known in the optics literature (Roggemann \& Welsh, 1996). For concreteness, we outline a derivation.

The zero-order Fourier component intensity is

$i_{0}(\boldsymbol{\varepsilon})=\left(\mathrm{a}_{\mathbf{0}}\right)^{2}=\left|\frac{1}{n^{2}} \sum_{\mathbf{s}} a_{\mathbf{s}} \mathrm{e}^{\mathrm{i} \varepsilon_{\mathbf{s}}}\right|^{2}$.

The Strehl ratio is

$S t(\boldsymbol{\varepsilon})=i_{0}(\boldsymbol{\varepsilon}) / i_{0}(\overline{\boldsymbol{\varepsilon}})$,

which is analogous to Eq. (9) if we identify $\left\{\varepsilon_{s}(k)\right\}$ in Eq. (33) with $[u(\mathbf{r}, t)+\phi(\mathbf{r})]$ in Eq. (9). The Strehl ratio given by Eq. (33) satisfies

$S t(\boldsymbol{\varepsilon})=1-\varphi(\varepsilon)+$ h.o.t.

To see this relationship between Strehl ratio and error covariance, simply take the Taylor series expansion of $i_{0}(\varepsilon)$ around $\bar{\varepsilon}$,

$$
\begin{aligned}
i_{0}(\boldsymbol{\varepsilon})= & i_{0}(\overline{\boldsymbol{\varepsilon}})+\left.\frac{\partial i_{0}}{\partial \boldsymbol{\varepsilon}}\right|_{\overline{\boldsymbol{\varepsilon}}} \cdot(\boldsymbol{\varepsilon}-\overline{\boldsymbol{\varepsilon}}) \\
& +\left.\frac{1}{2} \frac{\partial}{\partial \boldsymbol{\varepsilon}}\left(\frac{\partial i_{0}}{\partial \boldsymbol{\varepsilon}}\right)\right|_{\overline{\boldsymbol{\varepsilon}}} \cdot[(\boldsymbol{\varepsilon}-\overline{\boldsymbol{\varepsilon}}),(\boldsymbol{\varepsilon}-\overline{\boldsymbol{\varepsilon}})]+\cdots
\end{aligned}
$$

where $\overline{\boldsymbol{\varepsilon}}$ denotes the $n \times n$ matrix whose elements are all $\bar{\varepsilon}$. Computing the partial derivatives in Eq. (35) gives

$i_{0}(\boldsymbol{\varepsilon})=i_{0}(\overline{\boldsymbol{\varepsilon}})(1-\varphi(\boldsymbol{\varepsilon}))+$ h.o.t. 
There is thus a straightforward relationship between Strehl ratio maximization for the deterministic wave-front control problem and error covariance minimization for the wave-front estimation problem. The Strehl ratio can be measured using the Fourier-domain imager in Fig. 3, which raises the possibility of adapting the gain matrix on-line.

\subsection{Implications for system design}

Our general strategy for system design is then to start with the basic architecture of Fig. 1, and make good choices for the Fourier filter operator and the gain matrix. We have seen from the deterministic analysis that the proportional Fourier filter operator (with the peak phase shift constrained to be at most $\pi / 2$ ) has favorable properties. In the presence of noise, it is critical that the Fourier filter provide a filtered image with good SNR (i.e., good contrast), so that the system can start compensating phase distortion even when the initial distortion is severe.

The deterministic analysis of Section 3 places no constraints on the gain function (other than $\eta_{\min }<\eta(\mathbf{r})<\eta_{\max }$ for some positive constants $\eta_{\min }$ and $\eta_{\max }$ ). However, in the discrete-time setting, the dynamical Eq. (4) is replaced by a forward-Euler method, and it is evident that there is an upper limit on each element of the gain matrix. For linear estimation problems, the gains start out large, and decrease as the estimate is refined. A similar behavior is expected for the nonlinear estimator described above. Our strategy is then to make sure the gain matrix elements stay bounded appropriately (to ensure stability of the nonlinear system) when the estimation error is large, and to reduce the gain matrix (e.g., using a common scale factor) as the estimation error decreases. In the small-estimation-error regime, a linear approximation to the nonlinear estimator may be useful. By scaling the gain matrix based on the measured Strehl ratio, the system can transition naturally on its own from the nonlinear, large-distortion regime to the linear, small-distortion regime.

\section{Summary and conclusions}

Large arrays of sensors and actuators are becoming feasible to build. The challenge ahead is to devise effective control laws in conjunction with such arrays. In this paper we report on our results in meeting such a challenge within the context of optics-specifically, feedback control to correct optical wave-front distortions caused by atmospheric turbulence. We have discussed both architectures and algorithms that exploit a modern realization of the Zernike wave-front sensor.

In considering optical wave-front measurement and control, nonlinearity enters in an intrinsic way. We use the continuum limit of actuation to represent the control laws via semilinear partial differential equations, which in certain instances give rise to gradient dynamics. In this paper we

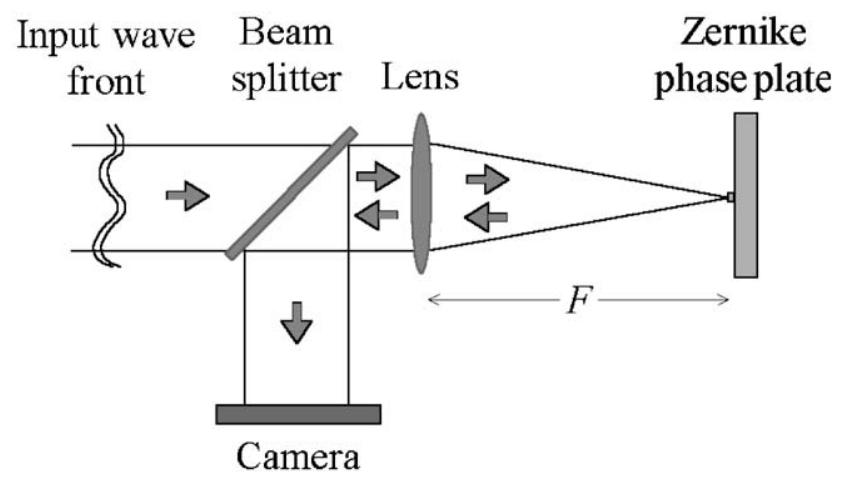

Fig. 6. Conventional Zernike wave-front sensor. The input beam is Fourier transformed by the lens, and the Zernike phase plate modulates the Fourier-domain complex envelope. The inverse-Fourier-transform intensity image is then captured by the camera. ( $F$ is the focal length of the lens.)

provide rigorous conclusions on the asymptotic behavior of such dynamics and also relate these results to experiments reported in companion papers. It is noteworthy that the control laws we describe admit parallel distributed implementations and hence scale well with the number of actuator elements.

Other optimization-based approaches to adaptive optics have recently emerged in the literature (Barchers \& Ellerbroek, 2001; Chu, Pauca, Plemmons, \& Sun, 2000). We hope to explore, in the future, possible connections between our current work and these recent contributions.

\section{Acknowledgements}

The authors would like to thank Leonid Beresnev for providing the LCLV used in the experimental work and Gary Carhart for programming support in the experimental validation. The authors would also like to thank Ralph Etienne-Cummings (of Johns Hopkins University, Baltimore, MD) and Steve Serati and Teresa Ewing (of Boulder Nonlinear Systems, Inc., Lafayette, CO) for valuable discussions.

\section{Appendix A. Conventional Zernike sensor}

The phase-contrast technique of Zernike can be understood in terms of the Fourier-transforming property of lenses, which is one manifestation of optical diffraction (Goodman, 1996). Assume a monochromatic input beam, so that it can be described using a complex envelope representation. As shown in Fig. 6, one lens Fourier-transforms the input beam, and the Zernike phase-plate (a glass slide with a phase-shifting dot perfectly centered on the optical axis) phase shifts the zero-order Fourier component relative to the rest of the Fourier spectrum. Another lens (or the same lens if the Zernike phase plate is reflective, as in 
Fig. 6) then performs an inverse Fourier transform. The two parts of the beam with different Fourier-domain phase shifts then interfere to produce an intensity pattern which is a nonlinear measurement of the input beam phase. The nonlinearity is analogous to the sinusoidal nonlinearity of an interferometer, but in addition, there is nonlocal coupling arising from the fact that the "reference beam" is taken from the input beam. We refer to the system of Fig. 6 as the "conventional Zernike wave-front sensor."

A linearized description of (an idealized approximation to) the conventional Zernike wave-front sensor, valid for small-magnitude phase variations in the input beam, was used by Zernike (in the context of microscopy) and by later authors (in the context of adaptive optics for astronomy) (Hardy, 1978; Dicke, 1975). If the input beam has a uniform intensity distribution over its cross-section, the linearized conventional Zernike wave-front sensor output intensity distribution is the sum of a constant term and a term proportional to the input beam phase distribution. The conventional Zernike wave-front sensor has certain practical disadvantages. The advantages of replacing the Zernike phase plate with an SLM have led to more recent interest in phase-contrast wave-front sensing, and to more detailed analysis of phase-contrast sensors (Ivanov et al., 1992; Glückstad, 1995; Seward et al., 1999; Glückstad \& Mogensen, 2001).

\section{Appendix B. Proof of Proposition 1}

We use standard results from the theory of second-order parabolic PDE (Evans, 1998). Eq. (4) is a special case of the general parabolic initial boundary-value problem

$$
\begin{aligned}
& \frac{\partial u}{\partial t}+L u=h \quad \text { in } \Omega_{T}, \\
& u=g \quad \text { on } \Omega \times\{t=0\}, \\
& h: \Omega_{T} \rightarrow \mathbb{R} \quad \text { and } g: \Omega \rightarrow \mathbb{R} \text { given, }
\end{aligned}
$$

with periodic boundary conditions, where $\Omega$ is the domain for the transverse coordinates $\mathbf{r}=(x, y)$, and $\Omega_{T}=\Omega \times(0, T)$ for some fixed $T>0$. From Eq. (4), $L=-\eta l^{2} \Delta$, and is assumed to be uniformly elliptic in $(x, y)$.

The initial data $g$ is assumed to satisfy $g \in L^{2}(\Omega)$. Since $h(\mathbf{r}, t)=-\eta f(u+\phi)$ involves feedback, we cannot assume (but instead must verify) that $h \in L^{2}\left(\Omega_{T}\right)$, i.e., that

$$
\int_{0}^{T} \int_{\Omega}|f(u+\phi)|^{2} \mathrm{~d} \mathbf{r} \mathrm{d} t<\infty .
$$

For $f=f_{\text {common }}$ given by Eq. (10), we have

$$
\begin{gathered}
\int_{0}^{T} \int_{\Omega}|f(u+\phi)|^{2} \mathrm{~d} \mathbf{r} \mathrm{d} t \\
\quad=16 \sin ^{2} \theta
\end{gathered}
$$

$$
\begin{aligned}
& \times \int_{0}^{T} \int_{\Omega} \mid \sum_{\mathbf{p} \in I} \operatorname{Im}\left\{a \mathrm{e}^{\mathrm{i}(u+\phi-(2 \pi / \gamma) \mathbf{p} \cdot \mathbf{r})}\right. \\
& \left.\times \frac{1}{\gamma^{2}} \int_{\Omega} a \mathrm{e}^{\mathrm{i}(u+\phi-(2 \pi / \gamma) \mathbf{p} \cdot \hat{\mathbf{r}})} \mathrm{d} \hat{\mathbf{r}}\right\}\left.\right|^{2} \mathrm{~d} \mathbf{r} \mathrm{d} t \\
\leqslant & 16\left(\sin ^{2} \theta\right) N \\
& \times \sum_{\mathbf{p} \in I} \int_{0}^{T} \int_{\Omega} \mid \operatorname{Im}\left\{a \mathrm{e}^{\mathrm{i}(u+\phi-(2 \pi / \gamma) \mathbf{p} \cdot \mathbf{r})}\right. \\
& \left.\times \frac{1}{\gamma^{2}} \int_{\Omega} a \mathrm{e}^{\mathrm{i}(u+\phi-(2 \pi / \gamma) \mathbf{p} \cdot \hat{\mathbf{r}})} \mathrm{d} \hat{\mathbf{r}}\right\}\left.\right|^{2} \mathrm{~d} \mathbf{r} \mathrm{d} t \\
\leqslant & 16\left(\sin ^{2} \theta\right) N \\
& \times \sum_{\mathbf{p} \in I} \int_{0}^{T} \int_{\Omega} \mid a \mathrm{e}^{\mathrm{i}(u+\phi-(2 \pi / \gamma) \mathbf{p} \cdot \mathbf{r})} \\
& \times\left.\frac{1}{\gamma^{2}} \int_{\Omega} a \mathrm{e}^{\mathrm{i}(u+\phi-(2 \pi / \gamma) \mathbf{p} \cdot \hat{\mathbf{r}})} \mathrm{d} \hat{\mathbf{r}}\right|^{2} \mathrm{~d} \mathbf{r} \mathrm{d} t \\
\leqslant & 16\left(\sin ^{2} \theta\right) N \sum_{\mathbf{p} \in I} \int_{0}^{T} \int_{\Omega} a^{2} \\
& \times \int_{\Omega}\left|a \mathrm{e}^{\mathrm{i}(u+\phi)}\right|^{2} \mathrm{~d} \hat{\mathbf{r}} \mathrm{d} \mathbf{r} \mathrm{d} t \\
= & 16\left(\sin ^{2} \theta\right) T N^{2}\left(\int_{\Omega} a^{2} \mathrm{~d} \mathbf{r}\right)^{2},
\end{aligned}
$$

where $N$ is the number of elements in $I$. (The property that the total energy in a single Fourier component must be less than the total energy of a function follows from Parceval's Theorem, and was used in arriving at the final inequality.) We thus conclude that the assumption that $\int_{\Omega} a^{2} \mathrm{~d} \mathbf{r}<\infty$ ensures that $h \in L^{2}\left(\Omega_{T}\right)$. A similar calculation holds for $f=$ $f_{\text {arb }}-f_{\mathrm{o}}$ (see Eq. (14)).

Since all the necessary hypotheses are satisfied, we can simply invoke the standard results from the theory of second-order parabolic PDE to conclude that

$$
\begin{aligned}
& u \in L^{2}\left(0, T ; H_{\text {per }}^{1}(\Omega)\right), \\
& u \in L^{2}\left(0, T ; H_{\text {per }}^{2}(\Omega)\right) \cap L^{\infty}\left(0, T ; H_{\text {per }}^{1}(\Omega)\right) .
\end{aligned}
$$

(For (B.5) to hold, we need to further assume that the initial data satisfies $g \in H_{\text {per }}^{1}(\Omega)$.) This amount of regularity is sufficient for purposes of Propositions 2 and 3.

\section{References}

Barchers, J. D., \& Ellerbroek, B. L. (2001). Improved compensation of turbulence-induced amplitude and phase distortions by means of multiple near-field phase adjustments. Journal of the Optical Society of America A, 18(2), 399-411.

Chu, M. T., Pauca, V. P., Plemmons, R. J., \& Sun, X. (2000). A mathematical framework for the linear reconstructor problem in adaptive optics. Linear Algebra and its Applications, 316(1-3), $113-135$. 
Dicke, R. H. (1975). Phase-contrast detection of telescope seeing errors and their correction. The Astrophysical Journal, 198, 605-615.

Evans, L. C. (1998). Partial differential equations. Providence, RI: American Mathematical Society.

Ferwerda, H. A. (1994). Frits Zernike - life and achievements. SPIE proceedings, phase contrast and differential interference contrast imaging techniques and applications, Vol. 1846 (pp. 2-9).

Fried, D. L. (1998). Branch point problem in adaptive optics. Journal of the Optical Society of America A, 15(10), 2759-2768.

Glückstad, J. (1995). Adaptive array illumination and structured light generated by spatial zero-order self-phase modulation in a Kerr medium. Optics Communications, 120, 194-203.

Glückstad, J., \& Mogensen, P. C. (2001). Optimal phase contrast in common-path interferometry. Applied Optics, 40(2), 268-282.

Goodman, J. W. (1996). Introduction to Fourier optics. New York: McGraw-Hill.

Gruev, G., \& Etienne-Cummings, R. (2000). A programmable spatiotemporal image processor chip. Proceedings of the IEEE international symposium on circuits and systems, Vol. IV (pp. $325-328)$.

Hardy, J. W. (1978). Active optics: A new technology for the control of light. Proceedings of IEEE, 66(6), 651-697.

Horenstein, M., Bifano, T. G., Pappas, S., Perreault, J., \& Krishnamoorthy-Mali, R. (1999). Real time optical correction using electrostatically actuated MEMS devices. Journal of Electrostatics, 46, 91-101.

Ivanov, V. Yu., Sivokon, V. P., \& Vorontsov, M. A. (1992). Phase retrieval from a set of intensity measurements: Theory and experiment. Journal of the Optical Society of America A, 9(9), 1515-1524.

Justh, E. W., \& Krishnaprasad, P. S. (2001). Analysis of a high-resolution optical wavefront control system. Proceedings of the conference on information sciences and systems, Vol. 2 (pp. 718-723).

Justh, E. W., Krishnaprasad, P. S., \& Vorontsov, M. A. (2000a). Nonlinear analysis of a high-resolution optical wavefront control system, Proceedings of the 39th IEEE conference decision and control, IEEE, New York (pp. 3301-3306).

Justh, E. W., Vorontsov, M. A., Carhart, G. W., Beresnev, L. A., \& Krishnaprasad, P. S. (2000b). Adaptive wavefront control using a nonlinear Zernike filter. SPIE Proceedings, 4124, 189-200.

Justh, E. W., Vorontsov, M. A., Carhart, G. W., Beresnev, L. A., \& Krishnaprasad, P. S. (2001). Adaptive optics with advanced phase-contrast techniques: Part II. High-resolution wavefront control. Journal of the Optical Society of America A, 18(6), 1300-1311.

Kushner, H. J., \& Yin, G. G. (1997). Stochastic approximation algorithms and applications. New York: Springer.

Ljung, L., \& Soderstrom, T. (1983). Theory and practice of recursive identification. Cambridge: MIT Press.

Pepper, D. M., Gaeta, C. J., \& Mitchell, P. V. (1995). Real-time holography, innovative adaptive optics, and compensated optical processors using spatial light modulators. In U. Efron (Ed.), Spatial light modulator technology: Materials, devices, and applications. New York: Marcel Dekker (pp. 585-654).

Roddier, F. (1999). Adaptive optics in astronomy. New York: Cambridge University Press.

Roggemann, M. C., \& Welsh, B. (1996). Imaging through turbulence. Boca Raton: CRC Press.

Serati, S. A., Sharp, G. D., Serati, R. A., McKnight, D. J., \& Stockley, J. E. (1995). $128 \times 128$ analog liquid crystal spatial light modulator, Optical Pattern Recognition VI. Proceedings of SPIE, 2490, 378-387.

Seward, A., Lacombe, F., \& Giles, M. K. (1999). Focal plane masks in adaptive optics systems. SPIE Proceedings Vol. 3762, Adaptive Optics Systems and Technology, 283-293.

Pluta, M., \& Szyjer, M. (Eds.) (1994). SPIE Proceedings Vol. 1846, Phase contrast and differential interference contrast imaging techniques and applications.
Vorontsov, M. A., Justh, E. W., \& Beresnev, L. A. (2001). Adaptive optics with advanced phase-contrast techniques: Part I. High-resolution wavefront sensing. Journal of the Optical Society of America A, 18(6), 1289-1299.

Vorontsov, M. A., Kirakosyan, M. E., \& Larichev, A. V. (1991). Correction of phase distortion in a nonlinear interferometer with an optical feedback loop. Soviet Journal of Quantum Electronics, 21, $105-108$.

Vorontsov, M. A., Shmalhauzen, V. I., \& Koriabin, A. V. (1988). Controlling optical systems. Moscow: Nauka.

Zernike, F. (1955). How I discovered phase contrast. Science, 121, 345-349.

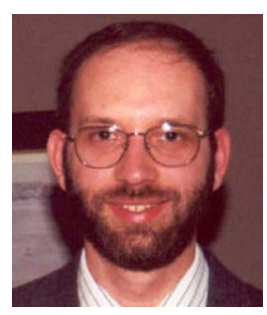

Eric Justh received a Ph.D. in Electrical Engineering from the University of Maryland, College Park, in 1998. From 1999 to 2001, he was a post-doc with the Institute for Systems Research at the University of Maryland, College Park, and since 2001 he has held an appointment as an Assistant Research Scientist with the Institute for Systems Research. His research interests are in applications for nonlinear dynamical systems, and projects he has worked on include pattern-forming systems, high-resolution adaptive optics, and (most recently) formation control for unmanned aerial vehicles.

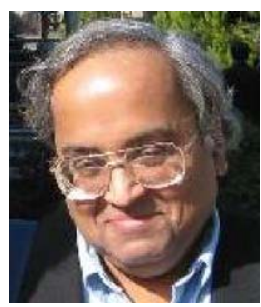

P. S. Krishnaprasad received his Ph.D. degree from Harvard University in 1977 . He was on the faculty of the Systems Engineering Department at Case Western Reserve University from 1977 to 1980 . He has been with the University of Maryland since August 1980, where he has held the position of Professor of Electrical Engineering since 1987, and a joint appointment with the Institute for Systems Research since 1988. He is also a member of the Applied Mathematics Faculty. He has held visiting positions with Erasmus University (Rotterdam); the Department of Mathematics, University of California, Berkeley; the University of Groningen (the Netherlands); the Mathematical Sciences Institute at Cornell University; and the Mechanical and Aerospace Engineering Department at Princeton University.

P. S. Krishnaprasad's research interests include problems of modeling, design, motion planning and control in mobile robotics; geometric methods in nonlinear dynamics; time-frequency analysis of acoustic signals and systems; intelligent control architectures, in part inspired by biological paradigms such as central patterns generators and neuronal networks; the technology and theory of smart materials such as piezo-electric and magnetostrictive materials for use in actuation and sensing; problems of high resolution optical wave front control; problems of integration of actuators and sensors in control networks.

P. S. Krishnaprasad is a Fellow of IEEE.

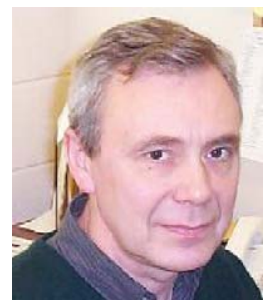

Mikhail A. Vorontsov received his Ph.D. in physics in 1977 and Doctor of Science in physics and mathematics in 1989, both from Moscow State University. Currently he is a senior physicist in the Computational and Information Sciences Directorate of the Army Research Laboratory and Director of the Intelligent Optics Laboratory at University of Maryland, College Park. He has published over 230 papers and four books on the subjects of adaptive optics, nonlinear spatio-temporal dynamics, imaging through turbulence, parallel image processing and correction. He is ARL, SPIE and OSA Fellow. 